\title{
Performance of JPEG Image Transmission Using Proposed Asymmetric Turbo Code
}

\author{
K. Ramasamy, ${ }^{1}$ Mohammad Umar Siddiqi, ${ }^{2}$ and Mohamad Yusoff Alias ${ }^{1}$ \\ ${ }^{1}$ Faculty of Engineering, Multimedia University, Cyberjaya 63100, Selangor, Malaysia \\ ${ }^{2}$ Faculty of Engineering, International Islamic University Malaysia, P.O. Box 10, Kuala Lumpur 50728, Malaysia
}

Received 23 February 2006; Revised 26 October 2006; Accepted 1 November 2006

Recommended by Richard J. Barton

This paper gives the results of a simulation study on the performance of JPEG image transmission over AWGN and Rayleigh fading channels using typical and proposed asymmetric turbo codes for error control coding. The baseline JPEG algorithm is used to compress a QCIF $(176 \times 144)$ "Suzie" image. The recursive systematic convolutional (RSC) encoder with generator polynomials $\left(1, D^{3}+D^{2}+1 / D^{3}+D+1\right)$, that is, $(13 / 11)$ in decimal, and $3 G$ interleaver are used for the typical WCDMA and CDMA2000 turbo codes. The proposed asymmetric turbo code uses generator polynomials $\left(1, D^{3}+D^{2}+1 / D^{3}+D+1 ; D^{3}+D^{2}+1 / D^{3}+1\right)$, that is, $(13 / 11 ; 13 / 9)$ in decimal, and a code-matched interleaver. The effect of interleaver in the proposed asymmetric turbo code is studied using weight distribution and simulation. The simulation results and performance bound for proposed asymmetric turbo code for the frame length $N=400$, code rate $r=1 / 3$ with Log-MAP decoder over AWGN channel are compared with the typical system. From the simulation results, it is observed that the image transmission using proposed asymmetric turbo code performs better than that with the typical system.

Copyright () 2007 Hindawi Publishing Corporation. All rights reserved.

\section{INTRODUCTION}

The constraints on bandwidth, power, and time in many image communication systems prohibit transmission of uncompressed raw image data. Compressed image representation, however, is very sensitive to bit errors, which can severely degrade the quality of the image at the receiver. A wireless channel generally suffers from severe effect of multipath propagation caused by the diffractions, reflections, and scattering from obstacles such as buildings, furniture, or moving objects. The transmitted signal arrives at the receiver from different paths, with each path introducing a time-varying attenuation and a time delay. The result is a set of replicas of the transmitted signal arriving at the receiver with time-varying amplitudes and phase shifts. Possible shadowing of the line-of-sight path by obstacles causes further variation of the received signal strength. The above problems make the channel a long burst-error channel. Thus, some error control strategy is needed to transmit highly compressed images reliably over such a burst-error channel to combat the effect of fading.

Turbo codes have attracted attention since introduced in 1993 [1]. Since turbo codes are a parallel concatenation of two or more convolutional codes separated by pseudo- random interleaver, the characteristic of both constituent encoder as well as the interleaver is important in order to achieve good performance. The parallel concatenated version of turbo codes introduced by Berrou et al. assumes identical component codes, hence known as symmetric turbo codes, which have either a good "waterfall" BER performance or a good "error floor" BER performance, but not both [1]. Several new classes of asymmetric turbo codes are introduced which improve performance compared to the original turbo code over the entire range of signal-to-noise ratios. In asymmetric turbo code, the first component code is chosen to obtain good performance in the waterfall region and the second component code is chosen to have a polynomial feedback which gives the overall turbo code a relatively high-weight code words. The resulting asymmetric turbo code provides a reasonable combination of performance at both a low and high SNR [2]. The parallel concatenation of a 16-state component code with a primitive feedback polynomial adopted by Perez et al. is known to lower the "error floor" compared to the Berrou code, but at a cost of poorer performance in the "waterfall" region [3]. The asymmetric turbo code used by Takeshita et al. adopted mixed type of component codes (different constraint length and/or defining polynomials). They adopted 16-state component codes with a particular 
kind of algebraic interleaver [4]. Massey et al. introduced a turbo code design using big numerator-little denominator (BN-LD) constituent codes, which increases the complexity of the feed forward portion of the impulse response and achieves improved performance in the waterfall region [5].

In this paper, we present simulation results on an image transmission system using a new class of asymmetric turbo codes [6], which consists of parallel concatenated convolutional codes with 8-state component codes (fixed constraint length), (13/11;13/9). The interleaver used is matched with the distance spectrum of the component code [6]. The paper is organized as follows: in Section 2, we present the proposed asymmetric turbo code. A simulation study is conducted to choose the best constituent code and interleaver and the performance results for various combinations of generator polynomials and a fixed random interleaver are provided. The effect of interleaver in the proposed asymmetric turbo code is also studied. Performance bound and simulation results for the typical and proposed asymmetric turbo codes on additive white Gaussian noise (AWGN) channel with frame size $N=400$ and code rate $r=1 / 3$ are compared in Section 3. Section 4 gives simulation results of an image transmission system over AWGN and Rayleigh fading channels using JPEG algorithm and typical turbo code and proposed turbo code as error control. Conclusions are given in Section 5.

\section{PROPOSED ASYMMETRIC TURBO CODE}

In typical turbo code system, a turbo encoder consists of two identical constituent RSC encoders with a pseudorandom interleaver preceding the second constituent encoder as shown in Figure 1. The turbo decoder also consists of two identical component decoders, and is illustrated in Figure 2. The performance of a turbo code may be affected by different parameters of the component codes, block size, interleaver design, and weight spectrum. This typical system results into few low-weight code words. However, we obtain more favorable distance spectrum by using a slightly different RSC encoder and a code-matched interleaver as shown in Figure 3; the corresponding decoding scheme is shown in Figure 4. In Figures 1 to 4, "I" and "DI" denote "interleaver" and "deinterleaver," respectively.

\subsection{RSC generator polynomial}

Generator polynomial of turbo encoder plays an important role in determining the weight of the code words [7]. To choose the best combination of generator polynomial for the modified turbo encoder, simulations were carried out for frame length, $N=400$ with RSC constraint length, $K=4$ and code rate, $r=1 / 3$. AWGN channel has been assumed with Log-MAP decoder with maximum number of iterations as 6 . Figure 5 shows the simulation results for various combinations of generator polynomial. The $E_{b} / N_{0}$ and BER values obtained with different generator polynomials are provided in Table 1 [6].

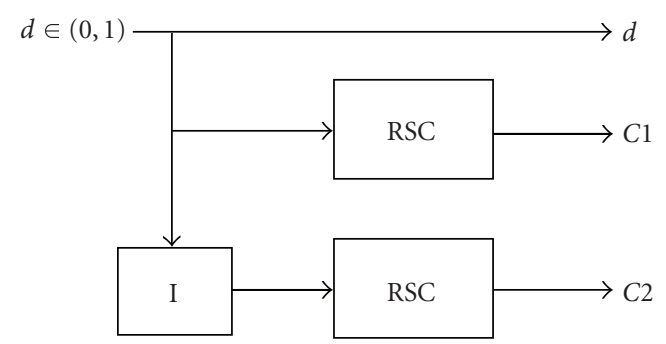

Figure 1: Typical turbo encoder.

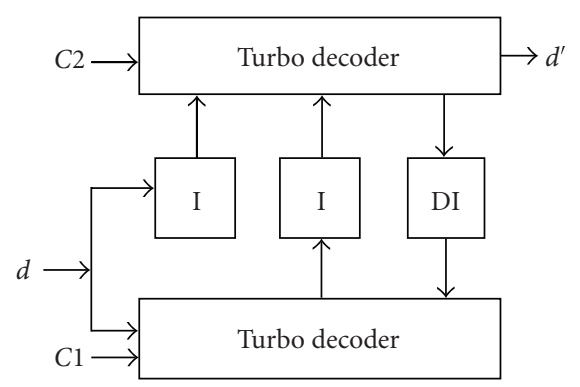

FIgURe 2: Typical turbo decoder.

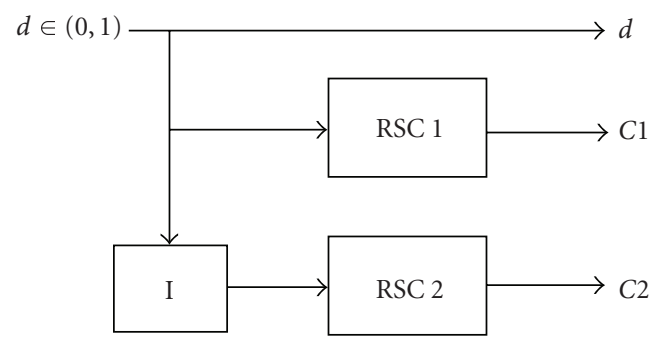

Figure 3: Proposed asymmetric turbo encoder.

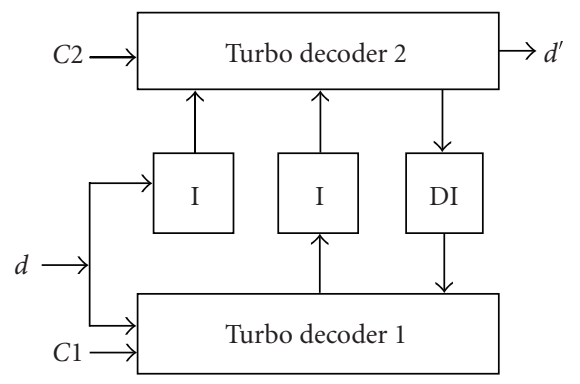

Figure 4: Proposed asymmetric turbo decoder. 


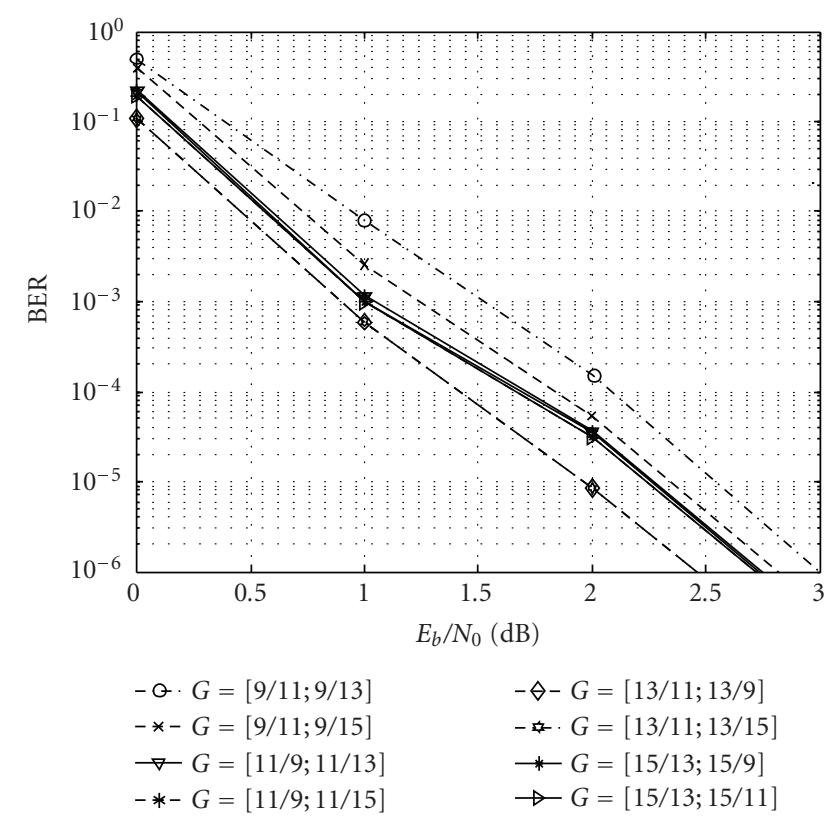

FIGURE 5: Simulation results for different generator polynomials.

It is noticed from Figure 5 and Table 1 that the generator polynomial $(13 / 11 ; 13 / 9)$ gives the best BER performance [6]. The maximum number of iterations required for various generator polynomial combinations is shown in Table 2. As shown in Table 2, although the generator polynomial $(13,11 ; 13,9)$ requires six iterations which is slightly higher than that for other combinations, the performance values are impressive. Therefore, there exists a trade-off between BER performance enhancement and delay increase due to iterations. Since the iteration difference between $(13,11 ; 13,9)$ and other generator polynomials does not exceed two, we choose $(13,11 ; 13,9)$ for our proposed asymmetric turbo encoder. The selection of generator polynomial is based on both better simulation results and improved weight spectrum as discussed in [8]. The analysis of the distance spectrum of proposed asymmetric turbo code for its improved performance is presented separately in [8].

\subsection{Interleaver}

The interleaver has a key role in shaping the weight distribution of the code, which ultimately controls its performance. So it is the most critical part in the design of a turbo code. A good interleaver design for a turbo code is the one, which produces high-weight output $[9,10]$. The complete weight spectra for several short block length proposed turbo codes are obtained. The algorithm for computing the turbo code free distance is based on the new notion of constrained sub codes, that is, a subset of a code defined via constraints on the edges of its trellis and permits the computation of large distances for large interleavers without a constraint on the in-

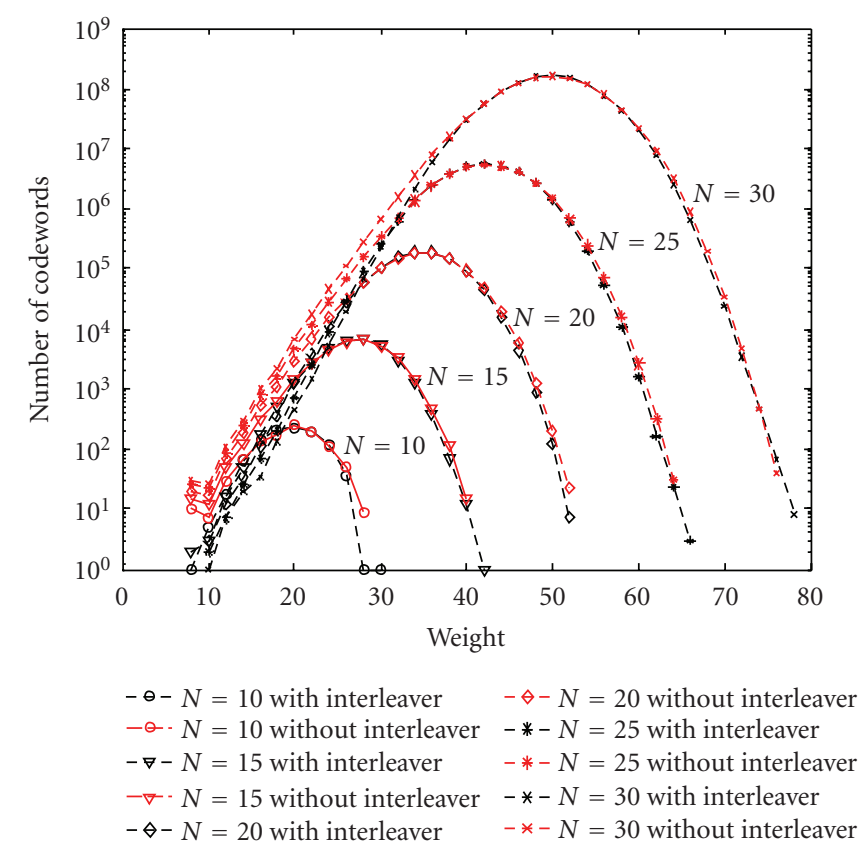

FIGURE 6: The effect of interleaver on weight distribution in proposed asymmetric turbo code.

put sequence weight [8]. Figure 6 shows the effect of random interleaver in the proposed asymmetric turbo code for the block size, $N=10,15,20,25$, and 30 bits [8]. It is observed that as the block size increases, the weight distribution improves. For the given block size, the weight distribution curve of turbo code with interleaver has a leading edge initially and lagging edge at the end, where as the turbo code without interleaver has lagging edge initially and leading edge at the end. Figure 7 shows the performance of proposed asymmetric turbo code over AWGN channel for the block length, $N=400, r=1 / 3$ with and without random interleaver. It is noticed that the interleaving gain is $1.5 \mathrm{~dB}$ at BER of $10^{-6}$. In some applications where the delay is crucial, the interleaver may be dropped at the cost of $E_{b} / N_{0}$ of $1.5 \mathrm{~dB}$. The design criteria of a code-matched interleaver used in proposed asymmetric code is provided in [6]. We eliminate low-weight code words with significant contributions to the error performance. The elimination of a specific code word can be done by breaking up the input pattern that generates that code word. The input information sequences with weights 2,3 , and 4 are considered in the interleaver design [6].

\section{PERFORMANCE BOUND AND SIMULATION RESULTS OF PROPOSED ASYMMETRIC TURBO CODE}

We define a uniform interleaver as a statistical device which maps a given input sequence of length $N$ and weight $w$ into all distinct $N_{c w}$ permutations of it with equal probability $1 / N_{c w}$. Making use of the properties of a uniform interleaver, the average conditional weight enumerate function (CWEF) 
TABLE 1: BER values for different generator polynomials.

\begin{tabular}{c|cccccccc}
\hline$E_{b} / N_{0}(\mathrm{~dB})$ & $(9,11 ; 9,13)$ & $(9,11 ; 9,15)$ & $(11,9 ; 11,13)$ & $(11,9 ; 11,15)$ & $(13,11 ; 13,9)$ & $(13,11 ; 13,15)$ & $(15,13 ; 15,9)$ & $(15,13 ; 15,11)$ \\
\hline 0 & $5.00 \mathrm{E}-01$ & $4.00 \mathrm{E}-01$ & $2.12 \mathrm{E}-01$ & $2.26 \mathrm{E}-01$ & $1.08 \mathrm{E}-01$ & $1.09 \mathrm{E}-01$ & $1.98 \mathrm{E}-01$ & $1.98 \mathrm{E}-01$ \\
1 & $8.00 \mathrm{E}-03$ & $2.57 \mathrm{E}-03$ & $1.02 \mathrm{E}-03$ & $1.16 \mathrm{E}-03$ & $6.00 \mathrm{E}-04$ & $6.02 \mathrm{E}-04$ & $9.87 \mathrm{E}-04$ & $9.87 \mathrm{E}-04$ \\
2 & $1.50 \mathrm{E}-04$ & $5.21 \mathrm{E}-05$ & $3.47 \mathrm{E}-05$ & $3.70 \mathrm{E}-05$ & $8.50 \mathrm{E}-06$ & $8.52 \mathrm{E}-06$ & $3.20 \mathrm{E}-05$ & $3.20 \mathrm{E}-05$ \\
3 & $9.68 \mathrm{E}-07$ & $4.07 \mathrm{E}-07$ & $2.70 \mathrm{E}-07$ & $2.84 \mathrm{E}-07$ & $7.80 \mathrm{E}-08$ & $7.82 \mathrm{E}-08$ & $2.48 \mathrm{E}-07$ & $2.48 \mathrm{E}-07$ \\
\hline
\end{tabular}

TABLE 2: Number of iterations for various generator polynomial combinations.

\begin{tabular}{l|c|c}
\hline RSC 1 & RSC 2 & Number of iterations \\
\hline$(9,11)$ & $(9,13)$ & 4 \\
$(9,11)$ & $(9,15)$ & 5 \\
$(11,9)$ & $(11,13)$ & 4 \\
$(11,9)$ & $(11,15)$ & 5 \\
$(13,11)$ & $(13,9)$ & 6 \\
$(13,11)$ & $(13,15)$ & 5 \\
$(15,13)$ & $(15,9)$ & 5 \\
$(15,13)$ & $(15,11)$ & 5 \\
\hline
\end{tabular}

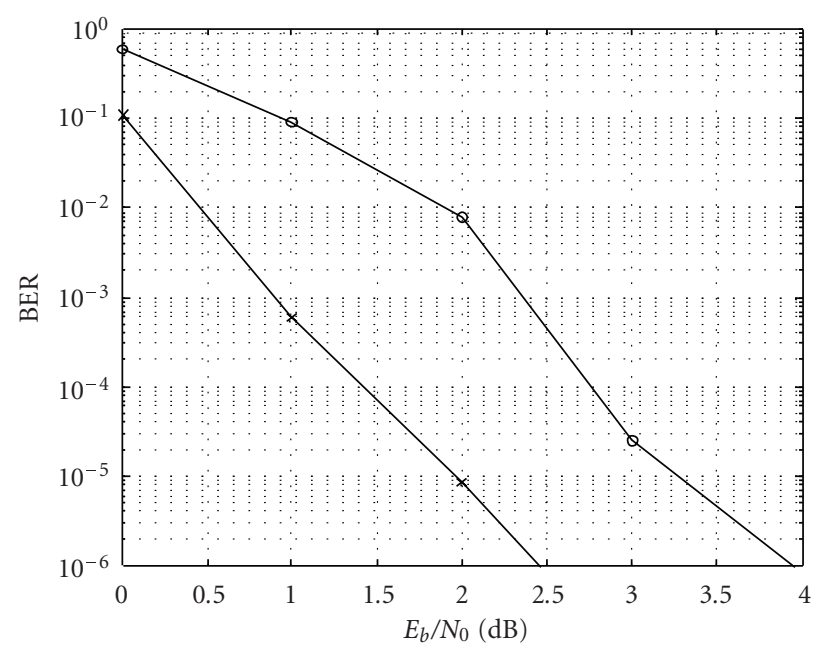

- Performance of proposed asymmetric turbo code without RI $\star$ Performance of proposed asymmetric turbo code with RI

FIGURE 7: Simulation results for proposed asymmetric turbo code with and without random interleaver (RI).

of all possible turbo codes with respect to the whole class of interleavers for turbo code system can be evaluated as given in (1) [11]:

$$
A_{w}^{T C}(Z)=\frac{A_{w}^{C_{1}}(Z) \cdot A_{w}^{C_{2}}(Z)}{N_{c w}}
$$

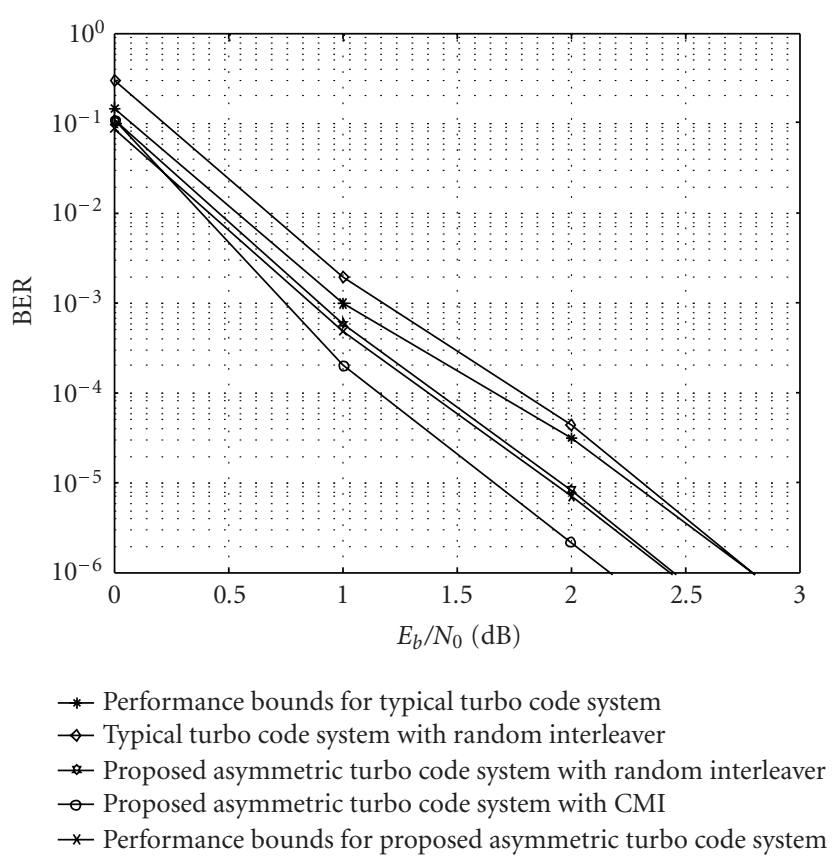

FIGURE 8: Performance bound and simulation results for typical and proposed asymmetric turbo code systems over AWGN channel, $N=$ $400, r=1 / 3$.

where $N_{c w}=(N / w)=N ! /(N-w) ! w !, A^{c_{1}}$ and $A^{c_{2}}$ are the weight enumerating functions for RSC1 and RSC2 encoders, respectively. Equation (1) represents an average turbo code with given constituent codes and block size $N$ over all possible interleavers. Here code words produced by both encoders are independent of each other, because $A^{c_{1}}$ and $A^{c_{2}}$ are assumed as individual components [12]. The average bit-error probability of the proposed asymmetric turbo code system over AWGN channel is evaluated by

$$
P_{\text {bit }} \leq \sum_{j} \sum_{w} \frac{w}{N} A_{w}^{T C}(Z) P_{2}(j),
$$

where $P_{2}(j)$ is the pairwise error probability between the all-zero codeword and codeword with minimum Hamming weight, $d$.

Figure 8 shows performance bound and simulation results of typical turbo code and proposed asymmetric turbo code for an information block length, $N=400, r=1 / 3$. AWGN channel has been assumed with Log-MAP decoder 


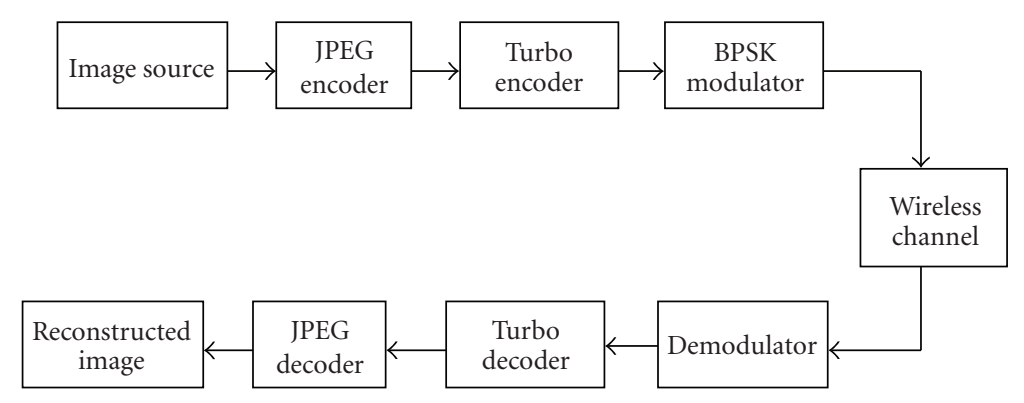

FIGURE 9: Image transmission system using typical and proposed turbo codes.

and the number of iteration is 6 . We notice that the proposed asymmetric turbo code performs better than typical turbo code and the coding gain is $0.6 \mathrm{~dB}$ at BER of $10^{-6}$. To verify the possibility of practical implementation of proposed turbo code, we simulated and compared the performance of typical turbo code and proposed asymmetric turbo code systems in $3 \mathrm{G}$ wireless communication standards: WCDMA and CDMA2000 [6]. The simulation results indicated that the performance of proposed asymmetric turbo code is superior to the performance of typical turbo code and the coding gain is from 0.5 to $0.8 \mathrm{~dB}$ for different channel conditions [6].

\section{IMAGE TRANSMISSION USING TYPICAL AND PROPOSED ASYMMETRIC TURBO CODES}

In this section, an image transmission system over AWGN and Rayleigh fading channels using typical and proposed asymmetric turbo codes as error control coding is provided. The baseline JPEG algorithm is used to compress a QCIF $(176 \times 144)$ "Suzie" image.

\subsection{The baseline JPEG image coding}

The implementation of JPEG algorithm in this work is based on the baseline sequential DCT based, which is lossy. At the input to the encoder, the source image samples will be grouped into $8 \times 8$ blocks. Then the elements will go through level shift, FDCT, quantization, zigzag, run length and DC encoding, and then the entropy encoding. Finally, a bit stream of compressed image data will be obtained at the end of the encoder. Decompression is the exact reverse process. To deal with synchronization problems due to channel errors for bit streams containing variable length codes, restart intervals are implemented during the encoding process by keeping track the size of each interval. The decoding process will be performed on each interval individually, instead of the whole stream of image data bits. Using this method, any error will be contained in the particular interval only, without propagating the error to subsequent data. After decoding an interval, the process will resynchronize and restart to decode the next interval.
TABLE 3: Reconstructed image quality using typical turbo code over AWGN channel.

\begin{tabular}{c|cl}
\hline Iteration & MSE & PSNR \\
\hline 1 & 1158.3 & 17.49 \\
2 & 626.57 & 20.16 \\
3 & 275.16 & 23.73 \\
4 & 21.058 & 34.9 \\
5 & 9.1 & 38.54 \\
\hline
\end{tabular}

\subsection{Simulation results of image transmission system}

Simulations are done to compress a QCIF $(176 \times 144)$ greylevel "Suzie" image for the quality factor of 68. The JPEG compressed data is then encoded using typical and proposed asymmetric turbo codes. BPSK modulation is used. The image transmission system is shown in Figure 9. After every iteration, the output of turbo decoder is given to the JPEG decoder to reconstruct the image and the decoded image is compared with the original to compute mean square error (MSE) and peak signal-to-noise ratio (PSNR) according to the following formula:

$$
\begin{gathered}
\mathrm{MSE}=\left(\sum_{i=1}^{M} \sum_{j=1}^{N}\left(f(x, y)-f^{\prime}(x, y)\right)^{2}\right) \times(M \times N)^{-1} . \\
\text { PSNR }=20 \log _{10}\left(\frac{255}{\mathrm{RMSE}}\right) .
\end{gathered}
$$

The original and the decoded "Suzie" images at the output of typical turbo code system over AWGN channel for iteration 1 to iteration 5 are shown in Figure 10. The $E_{b} / N_{0}$ is set as $2 \mathrm{~dB}$. As shown in Table 3, the MSE Therefore, a zero MSE value is achieved for identical images. Higher values denote higher deviation between the original and degraded images. Note that a low MSE does not necessarily indicate high subjective quality. PSNR is derived using the root mean square error (RMSE) to denote deviation of a compressed image from the original in $\mathrm{dB}$. For an eight-bit image, with 


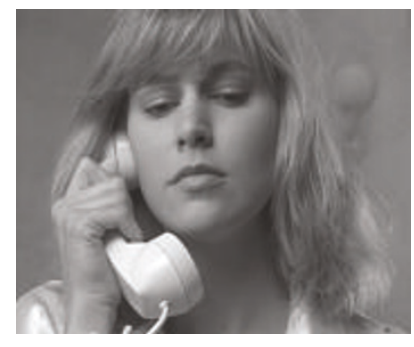

(a) Original

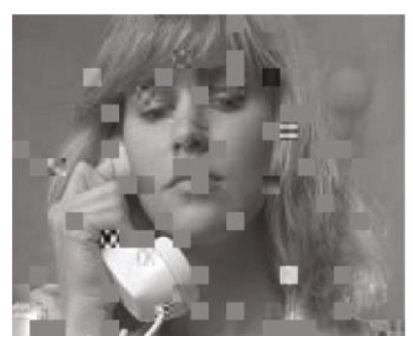

(d) Iteration 3

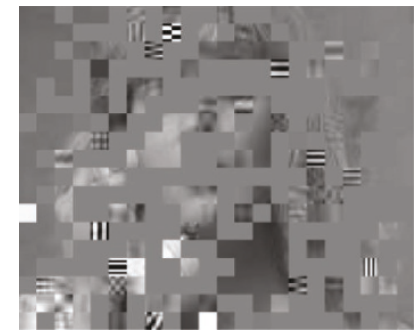

(b) Iteration 1

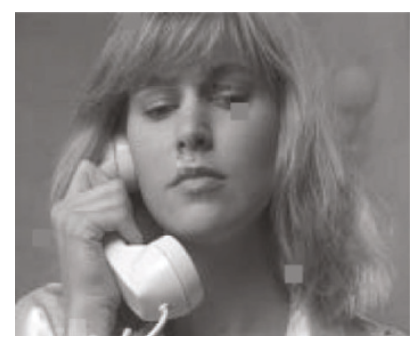

(e) Iteration 4

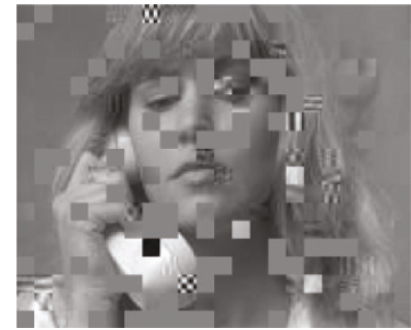

(c) Iteration 2

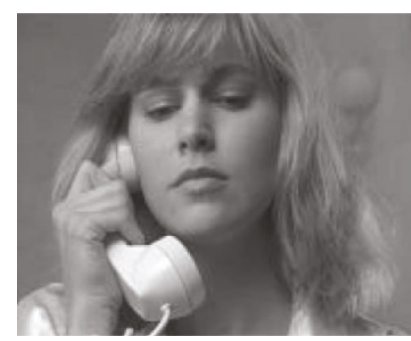

(f) Iteration 5

FIgURE 10: Original and decoded "Suzie" images over AWGN channel using typical turbo code with an $E_{b} / N_{0}$ of $2 \mathrm{~dB}$.

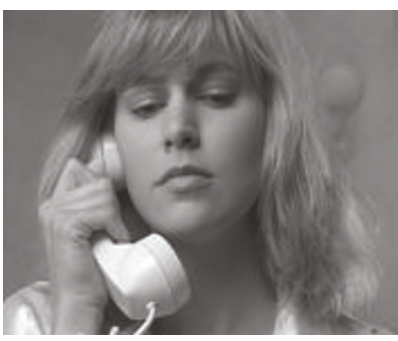

(a) Original

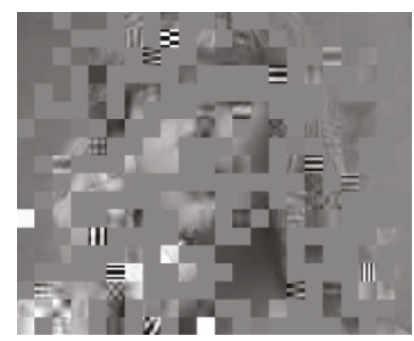

(b) Iteration 1

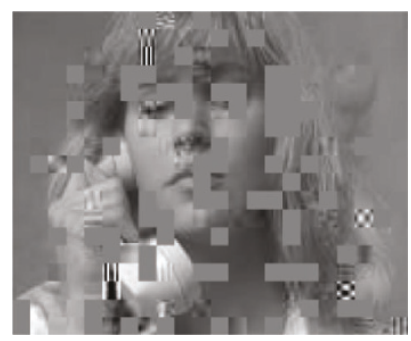

(c) Iteration 2

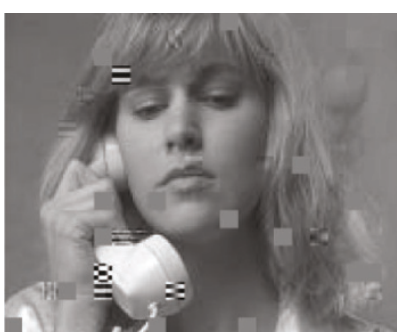

(d) Iteration 3

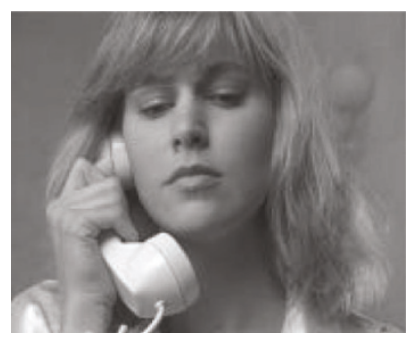

(e) Iteration 4

FIGURE 11: Original and decoded "Suzie" images over AWGN channel using proposed asymmetric turbo code with interleaver with an $E_{b} / N_{0}$ of $2 \mathrm{~dB}$.

intensity values between 0 and 255, the PSNR is given by decreases and PSNR increases as we increase the iteration. It is also noticed that even after 5th iteration, MSE of 9.1 is left uncorrected, which conforms that baseline JPEG is lossy. The original and the decoded "Suzie" images at the output of proposed asymmetric turbo code system over AWGN channel are shown in Figure 11. It is observed that it requires only four iterations to correct the errors where as typical turbo code requires five iterations. The quality of the reconstructed images for every iteration is provided in Table 4 . The decoded 


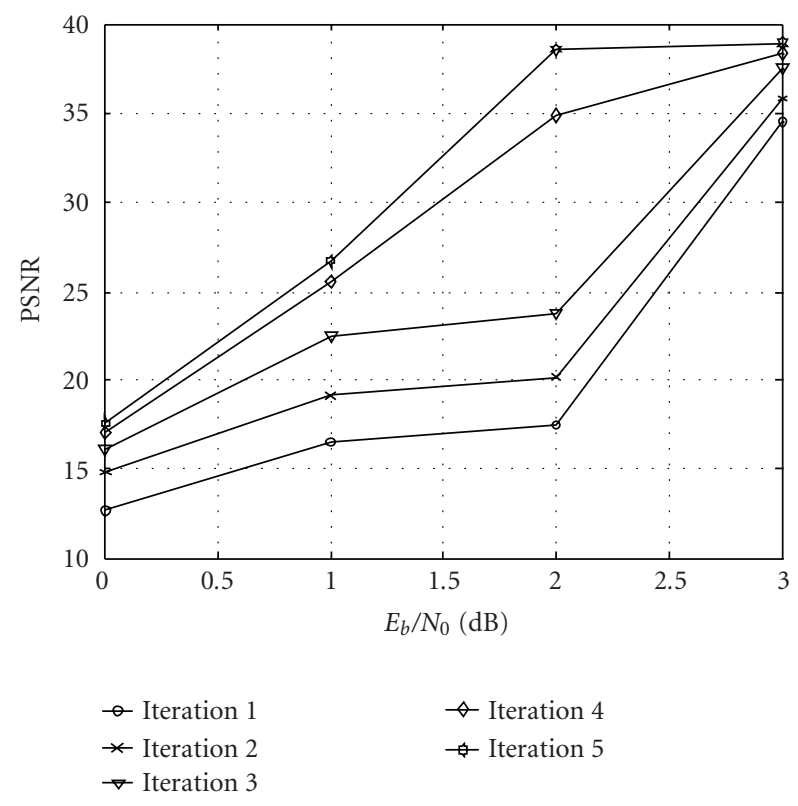

FIGURE 12: Decoded image quality (in PSNR) of typical turbo code over AWGN channel.

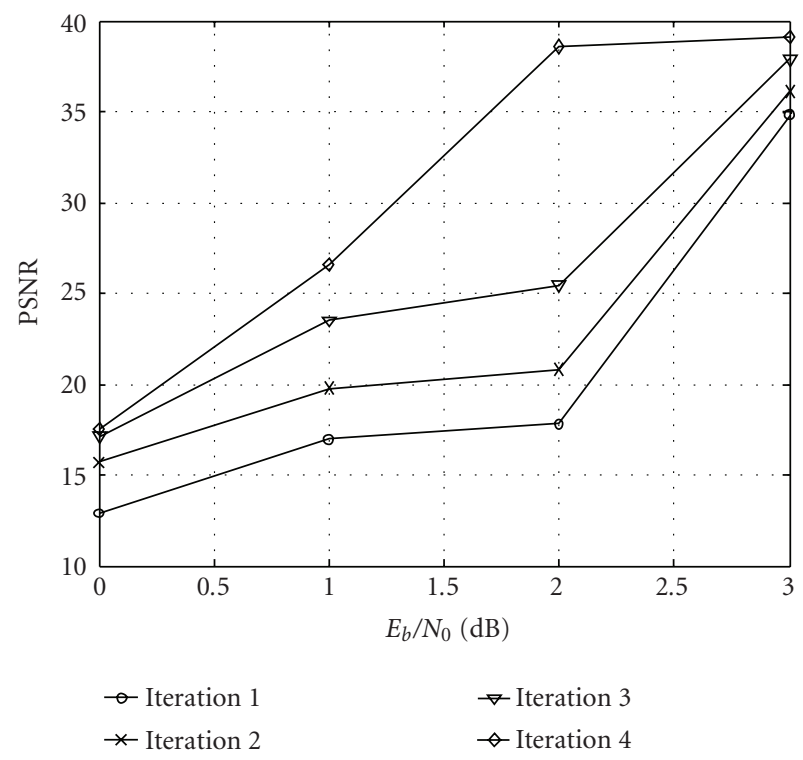

FIgURE 13: Decoded image quality (in PSNR) of proposed asymmetric turbo code with inetrleaver over AWGN channel.

image quality (in PSNR) of typical turbo code and the proposed turbo code systems over AWGN channel are also provided in Figures 12 and 13, respectively. We observe that higher performance gains are achieved using proposed asymmetric turbo code for all iterations and there is no increase in gain after the fourth iteration. The original and the decoded "Suzie" images at the output of proposed asymmetric turbo code system without interleaver over AWGN channel
TABLE 4: Reconstructed image quality using proposed asymmetric turbo code over AWGN channel.

\begin{tabular}{c|cc}
\hline Iteration & MSE & PSNR \\
\hline 1 & 1081.8 & 17.79 \\
2 & 546.71 & 20.75 \\
3 & 188.05 & 25.39 \\
4 & 9.1 & 38.54 \\
\hline
\end{tabular}

TABLE 5: Reconstructed image quality using proposed asymmetric turbo code without interleaver over AWGN channel.

\begin{tabular}{c|cl}
\hline Iteration & MSE & PSNR \\
\hline 1 & 1169.5 & 17.45 \\
2 & 878.15 & 18.7 \\
3 & 679.52 & 19.81 \\
4 & 452.87 & 21.57 \\
5 & 229.78 & 24.52 \\
6 & 69.297 & 29.72 \\
7 & 9.1 & 38.54 \\
\hline
\end{tabular}

are shown in Figure 14. It is observed that it requires seven iterations to correct the errors where as the proposed asymmetric turbo code with interleaver requires only four iterations. Thus, if the delay is crucial, the interleaver may be dropped. The quality of the reconstructed images for every iteration is provided in Table 5 . The decoded image quality (in PSNR) of the proposed turbo code system without interleaver over AWGN channel is also provided in Figure 15. We notice that only slight performance gains are achieved using the proposed turbo code without interleaver for every iteration. The original and the decoded "Suzie" images at the output of typical and proposed asymmetric turbo code systems over Rayleigh fading channel are shown in Figures 16 and 17 , respectively. The $E_{b} / N_{0}$ is set as $6 \mathrm{db}$ and $f_{d}=185 \mathrm{~Hz}$. It is observed that typical code requires eight iterations to correct the errors where as proposed asymmetric turbo code requires only seven iterations. The quality of the reconstructed images at the output of typical and proposed asymmetric turbo code systems for every iteration is provided in Tables 6 and 7, respectively. The decoded image quality (in PSNR) of typical turbo code and the proposed turbo code systems over AWGN and Rayleigh fading channels are also compared in the Figure 18. We notice that the performance of proposed asymmetric turbo code over AWGN channel with 4 iterations is same as that of the typical turbo code with 5 iterations. It is also observed that the performance gain of proposed asymmetric turbo code over Rayleigh fading channel with 7 iterations is higher or at least equal to that of the typical turbo code with 8 iterations.

\section{CONCLUSIONS}

In this paper, we presented the results of a study on the performance of an image transmission system using typical 


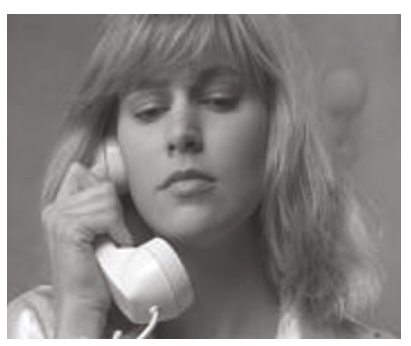

(a) Original

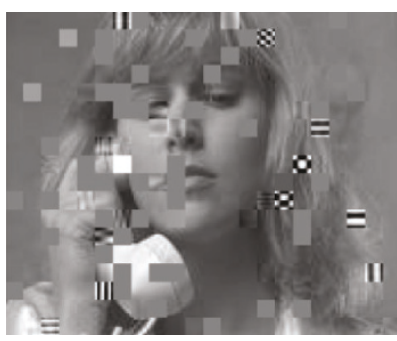

(e) Iteration 4

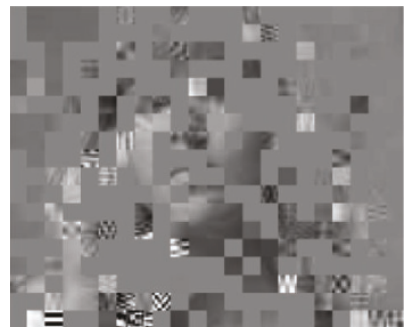

(b) Iteration 1

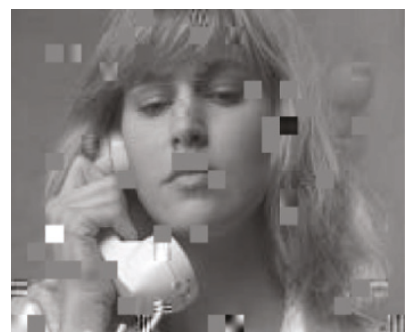

(f) Iteration 5

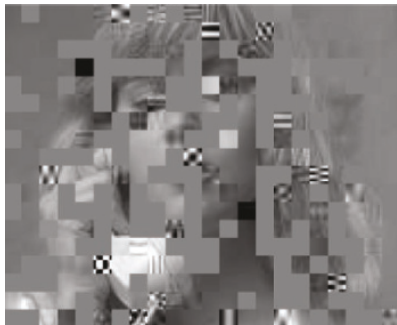

(c) Iteration 2

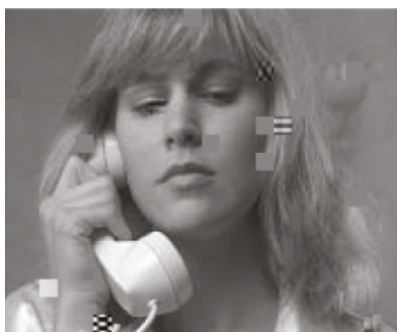

(g) Iteration 6

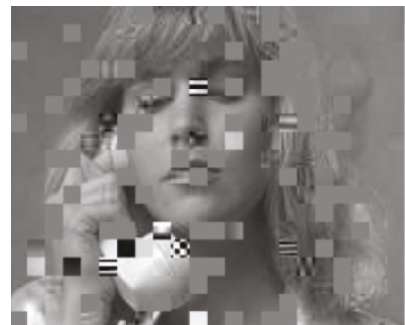

(d) Iteration 3

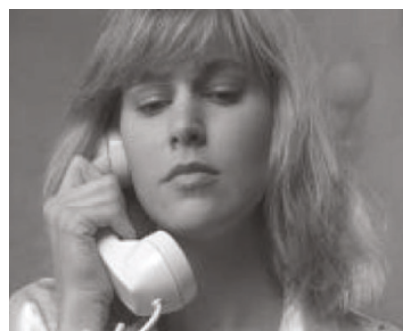

(h) Iteration 7

FIGURE 14: Original and decoded "Suzie" images over AWGN channel using proposed asymmetric turbo code without interleaver with an $E_{b} / N_{0}$ of $2 \mathrm{~dB}$.

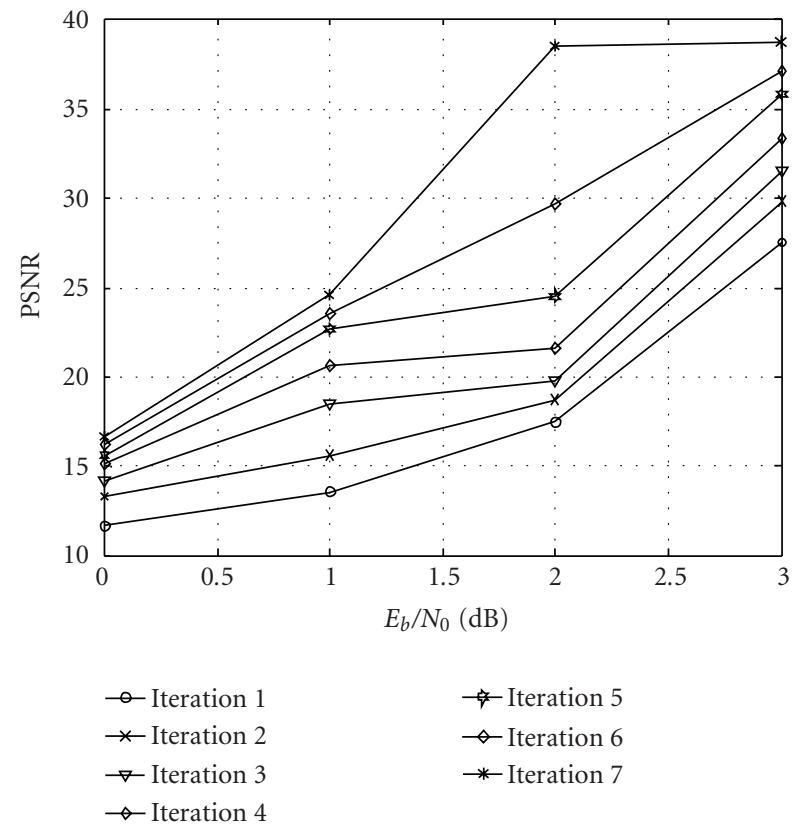

FIGURE 15: Decoded image quality (in PSNR) of proposed asymmetric turbo code without interleaver over AWGN channel.

and proposed asymmetric turbo codes. Although the search procedure of perfect parameters for good component encoder at low and high SNR is quiet exhaustive, the modifications in turbo encoder really contribute performance improvements in turbo code system. The simulation results in-
TABLE 6: Reconstructed image quality using typical turbo code over Rayleigh fading channel.

\begin{tabular}{c|cl}
\hline Iteration & MSE & PSNR \\
\hline 1 & 1465.1 & 16.47 \\
2 & 1286.9 & 17.04 \\
3 & 1066.4 & 17.85 \\
4 & 878.15 & 18.7 \\
5 & 559.22 & 20.65 \\
6 & 178.12 & 25.62 \\
7 & 57.781 & 30.51 \\
8 & 9.1008 & 38.54 \\
\hline
\end{tabular}

TABLE 7: Reconstructed image quality using proposed asymmetric turbo code over Rayleigh fading channel.

\begin{tabular}{c|cc}
\hline Iteration & MSE & PSNR \\
\hline 1 & 1369.9 & 16.76 \\
2 & 1168.9 & 17.45 \\
3 & 921.11 & 18.49 \\
4 & 793.72 & 19.13 \\
5 & 540.89 & 20.8 \\
6 & 115.76 & 27.5 \\
7 & 9.1 & 38.54 \\
\hline
\end{tabular}

dicate that the performance of image transmission system using proposed asymmetric turbo code is superior to that using typical turbo code for different channel conditions. 


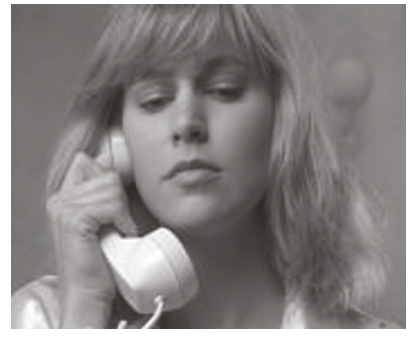

(a) Original

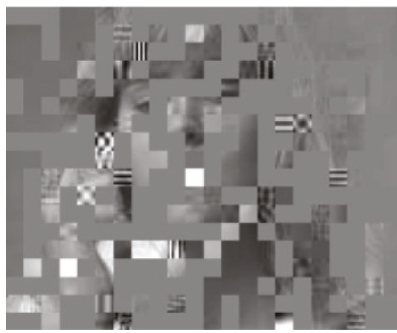

(d) Iteration 3

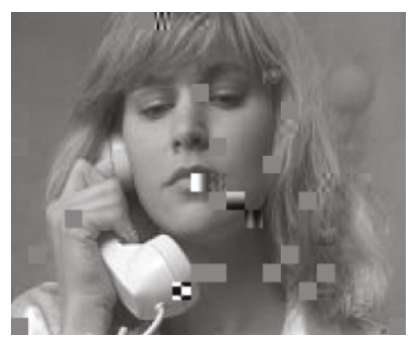

(g) Iteration 6

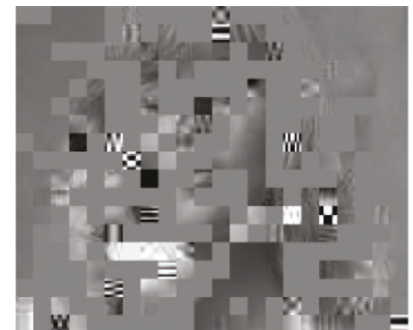

(b) Iteration 1

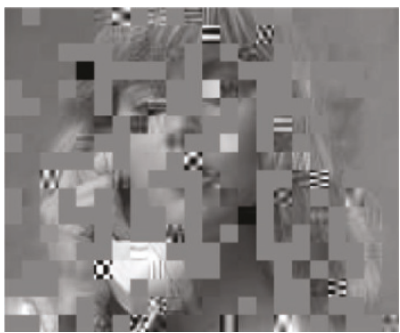

(e) Iteration 4

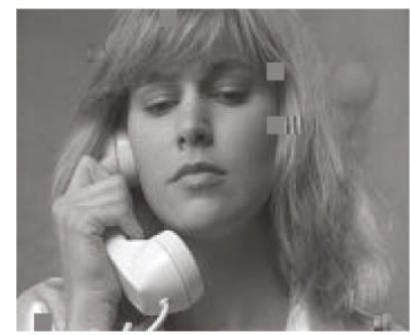

(h) Iteration 7

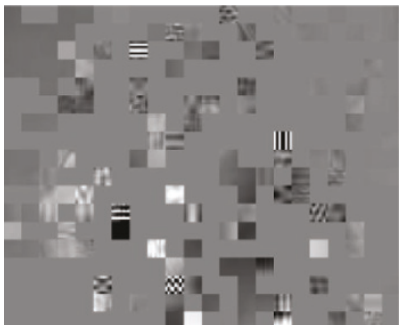

(c) Iteration 2

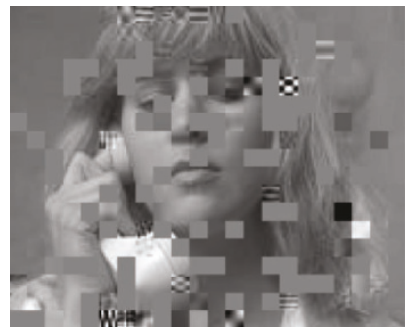

(f) Iteration 5

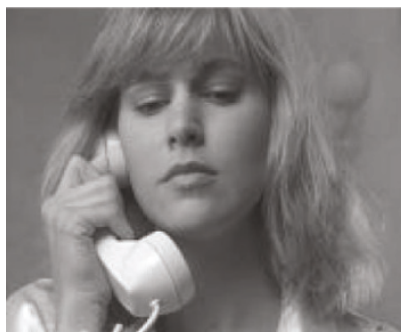

(i) Iteration 8

FIgURE 16: Original and decoded "Suzie" images over Rayleigh fading channel using typical turbo code with an $E_{b} / N_{0}$ of $6 \mathrm{~dB}, f_{d}=185 \mathrm{~Hz}$.

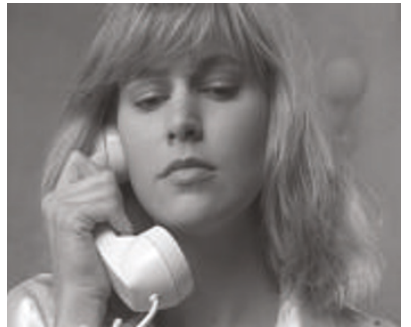

(a) Original

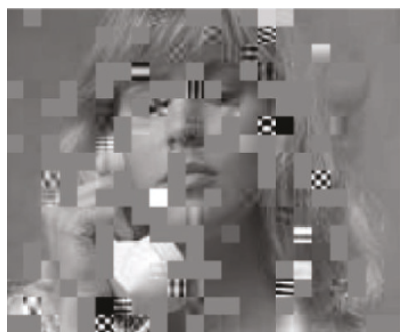

(e) Iteration 4

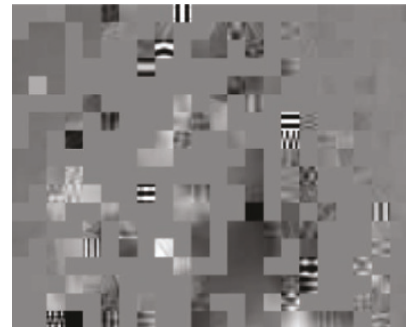

(b) Iteration 1

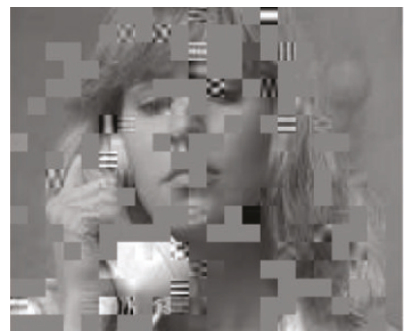

(f) Iteration 5

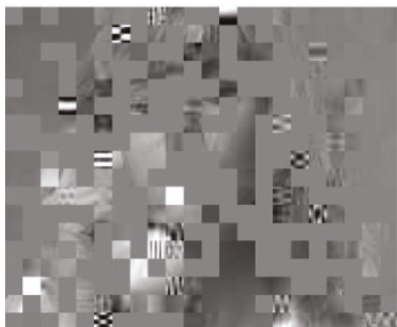

(c) Iteration 2

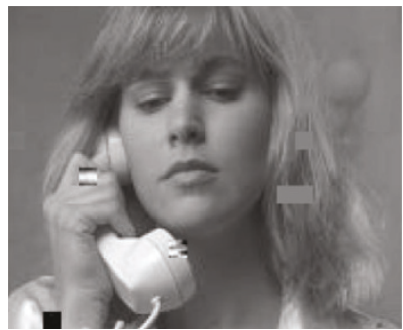

(g) Iteration 6

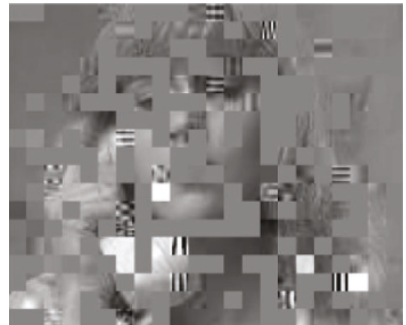

(d) Iteration 3

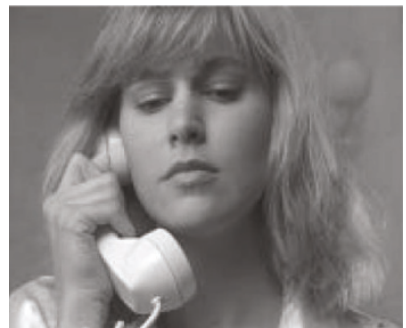

(h) Iteration 7

FigURE 17: Original and decoded "Suzie" images over Rayleigh fading channel using proposed asymmetric turbo code with an $E_{b} / N_{0}$ of $6 \mathrm{~dB}$, $f_{d}=185 \mathrm{~Hz}$. 


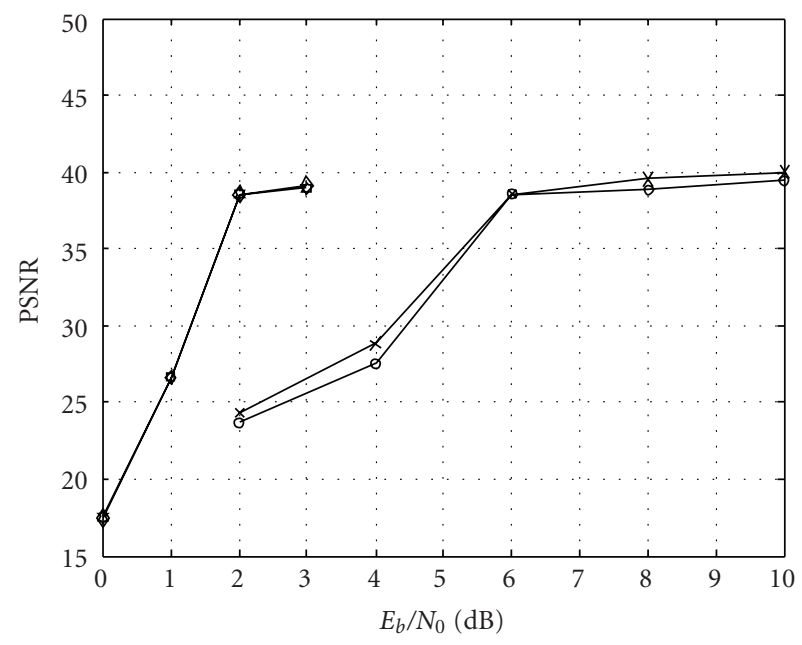

ॠ Typical turbo code over AWGN (5 iterations)
$\rightarrow$ Proposed asymmetric turbo code over AWGN (4 iterations)
$\rightarrow$ Typical turbo code over Reyleigh ( 8 iterations)
* Proposed asymmetric turbo code over Reyleigh ( 7 iterations)

FIGURE 18: Comparison of decoded image quality (in PSNR) of typical turbo code and proposed asymmetric turbo code systems.

\section{REFERENCES}

[1] C. Berrou, A. Glavieux, and O. Thitimajshima, "Near Shannon limit error-correcting coding and decoding: turbo-codes. 1," in Proceedings of International Conference on Communication (ICC '93), vol. 2, pp. 1064-1070, Geneva, Switzerland, May 1993.

[2] M. C. Valenti and J. Sun, "Turbo codes," in Handbook of RF and Wireless Technologies, chapter 12, pp. 375-400, Newnes, Oxford, UK, 2004.

[3] L. C. Perez, J. Seghers, and D. J. Costello Jr., "A distance spectrum interpretation of turbo codes," IEEE Transactions on Information Theory, vol. 42, no. 6, part 1, pp. 1698-1709, 1996.

[4] O. Y. Takeshita, O. M. Collins, P. C. Massey, and D. J. Costello Jr., "A note on asymmetric turbo-codes," IEEE Communications Letters, vol. 3, no. 3, pp. 69-71, 1999.

[5] P. C. Massey, O. Y. Takeshita, and D. J. Costello Jr., "Contradicting a myth: good turbo codes with large memory order," in Proceedings of IEEE International Symposium on Information Theory, p. 122, Sorrento, Italy, June 2000.

[6] K. Ramasamy, B. Balakrishnan, and M. U. Siddiqi, "A new class of asymmetric turbo code for $3 \mathrm{G}$ systems," AEU - International Journal of Electronics and Communications, vol. 60, no. 6, pp. 447-458, 2006.

[7] J. D. Andersen, "Selection of component codes for turbo coding based on convergence properties," Annales des Telecommunications, vol. 54, no. 3, pp. 208-213, 1999, special issue on iterated decoding.

[8] K. Ramasamy and M. U. Siddiqi, "Weight distribution analysis of proposed asymmetric turbo code for improved performance," AEU - International Journal of Electronics and Communications, vol. 60, no. 7, pp. 479-493, 2006.

[9] J. D. Andersen and V. V. Zyablov, "Interleaver design for turbo coding," in Proceedings of International Symposium on Turbo Codes, pp. 154-156, Brest, France, September 1997.
[10] D. Divsalar and F. Pollara, "On the design of turbo codes," The Telecommunications and Data Acquisition Progress (TDA) Progress Report 42-123, pp. 99-121, Jet Propulsion Laboratory (JPL), Pasadena, Calif, USA, November 1995.

[11] S. Benedetto and G. Montorsi, "Unveiling turbo codes: some results on parallel concatenated coding schemes," IEEE Transactions on Information Theory, vol. 42, no. 2, pp. 409-428, 1996.

[12] D. Divsalar, S. Dolinar, F. Pollara, and R. J. McEliece, "Transfer function bounds on the performance of turbo codes," The Telecommunications and Data Acquisition Progress (TDA) Progress Report 42-122, pp. 44-55, Jet Propulsion Laboratory (JPL), Pasadena, Calif, USA, August 1995.

K. Ramasamy was born in Sivakasi, India, on March 10, 1966. He received the B.Engg. degree in electronics and communication engineering from Madurai Kamaraj University, India, the M.Engg. degree in applied electronics from Bharathiar University, India, and the Ph.D. degree from Multimedia University, Malaysia, in 1988, 1993, and 2006, respectively. He joined the Faculty of V.L.B. Janakiammal College of Engineering and Technology, Coimbatore, India, in July 1988. From July 1988 to July 2001, he served as Associate Lecturer, Lecturer, Senior Lecturer, and Assistant Professor. In 2001, he joined as a Lecturer the Faculty of Engineering at Multimedia University, Malaysia. He has published more than 20 papers in international journals and conferences. His research interests include error-correcting codes and wireless communications.

Mohammad Umar Siddiqi received the B.S. Engg. and M.S. Engg. degrees from Aligarh Muslim University (AMU, Aligarh) in 1966 and 1971, respectively, and the Ph.D. degree from Indian Institute of Technology Kanpur (IIT Kanpur) in 1976, all in electrical engineering. He has been in the teaching profession throughout, first at AMU Aligarh, then at IIT Kanpur. In 1998, he joined Multimedia University, Malaysia. Currently, he

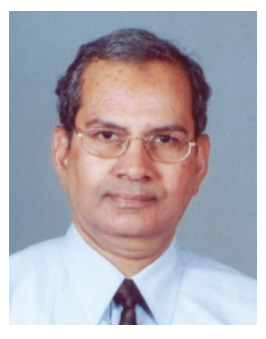
is a Professor in the Faculty of Engineering at International Islamic University Malaysia. He has published more than 100 papers in international journals and conferences. His research interests are in error-control coding, cryptography, and information security.

Mohamad Yusoff Alias obtained the B.S. degree in engineering (electrical engineering) from the University of Michigan, Ann Arbor, in May 1998. He then received his Ph.D. degree in December 2004 from the School of ECS, University of Southampton in the United Kingdom. He is currently a Lecturer in the Faculty of Engineering, Multimedia University in Malaysia. His research interests cover the field of wireless commu-

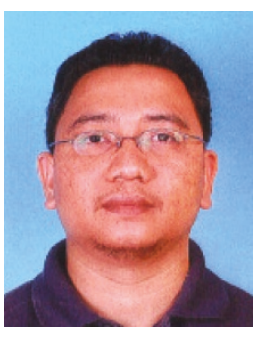
nications, especially in OFDM, multiple-antenna systems, multiuser detection, genetic algorithms in communications, and multimedia applications. 